\title{
Thymic myoid cells protect thymocytes from apoptosis and modulate their differentiation: implication of the ERK and Akt signaling pathways
}

\author{
R Le Panse ${ }^{\star, 1}$ and S Berrih-Aknin ${ }^{1}$ \\ ${ }^{1}$ CNRS UMR 8078, IPSC, Université Paris XI, Hôpital Marie Lannelongue, \\ Le Plessis-Robinson, France \\ * Corresponding author: R Le Panse, CNRS UMR 8078, IPSC, Université Paris \\ XI, Hôpital Marie Lannelongue, Le Plessis-Robinson, France. \\ Tel: + 330140942800 ext 35 65; Fax: + 3301463045 64; \\ E-mail: rozen.lepanse @ccml.u-psud.fr
}

Received 06.12.04; accepted 10.1.05; published online 18.3.05

Edited by RA Knight

\begin{abstract}
Thymic myoid cells correspond to a muscle-like cell population present in the thymic medulla. They are well conserved throughout species evolution, but their biological role is not known. We demonstrated that myoid cells protected thymocytes from apoptosis as evidenced by a strong decrease of annexin-V-FITC positive thymocytes. This effect was (1) specific of myoid cells compared to thymic epithelial cells; (2) dependent on direct cell-to-cell contacts and (3) triggered rapidly after $2 \mathrm{~h}$ in cocultures. This protective phenomenon was due to the activation of prosurvival mechanisms. Indeed, myoid cells activated extracellular-regulated kinases (ERK1/2) and Akt in thymocytes. Myoid cells also influenced thymocyte maturation. We observed an increase in $\mathrm{CD}^{+}$and a decrease in $\mathrm{CD}^{+}$single positive (SP) thymocytes when cocultured with myoid cells, independently of a $\mathrm{CD8}{ }^{+} \mathrm{SP}$ increased death or a CD4 ${ }^{+}$SP overproliferation. Consequently, thymic myoid cells protect thymocytes from apoptosis and could also modulate their differentiation process.

Cell Death and Differentiation (2005) 12, 463-472.

doi:10.1038/sj.cdd.4401611

Published online 18 March 2005
\end{abstract}

Keywords: muscle-like cells; thymocytes; survival; differentiation; thymus

\begin{abstract}
Abbreviations: MITC, myoid immortalized thymic cells; TEC thymic epithelial cells; MAPK, mitogen-activated protein kinase; ERK, extracellular-regulated kinase; PI3K, phosphatidylinositol 3-kinase; MHC, self-major histocompatibility complex; DN, double negative; DP, double positive; SP, single positive; TCR, T-cell receptor
\end{abstract}

\section{Introduction}

The thymus provides a complex environment essential for T-cell development. Differentiation of T cells occurs while they are progressing through the different thymus compartments. In their first differentiation steps, immature T-cell precursors become progressively double positive (DP) for CD4 and CD8 coreceptors and acquire a complete T-cell receptor (TCR). Further successful differentiation of thymocytes depends on the quality and the specificity of the interaction of their TCR with selfmajor histocompatibility complex (MHC). Only a few DP thymocytes pass successfully positive selection and are exported out of the thymus. The large majority of thymocytes die either because the TCR-MHC interaction is too weak (death by neglect) or, in contrast, because the TCR-MHC interaction is too strong (negative selection, self-tolerance). Positively selected DP thymocytes end up single positive (SP) cells for either CD4 or CD8 (lineage commitment): DP thymocytes expressing $\mathrm{MHC}$ class II specific TCR differentiate into CD4 ${ }^{+}$SP (T helper cells) whereas those expressing MHC class I specific TCR differentiate into $\mathrm{CD}^{+} \mathrm{SP}$ (T cytotoxic cells). ${ }^{1}$

Different signaling pathways modulate apoptosis versus survival balance in thymocytes and, consequently, control their differentiation process. Akt is a serine/threonine kinase, playing a prominent role in cell survival. ${ }^{2}$ In lymphocytes, Akt is activated by diverse extracellular signals such as cytokines, TCR signaling and CD28. ${ }^{2,3}$ Mitogen-activated protein kinase (MAPK) cascades play also an important role in inducing either apoptosis or cell survival. The cJun NH2-terminal kinase/stress-activated protein kinase (JNK/SAPK) ${ }^{4}$ and the p38 MAPK cascades $^{5}$ are mainly activated by cellular stresses and their activation is generally associated with apoptosis. ${ }^{6}$ In contrast, the extracellular-regulated kinase (ERK) cascade, involving ERK1 and ERK2, is especially involved in cell proliferation and also in cell survival. ${ }^{6,7}$ Moreover, in thymocytes, the ERK signaling pathway regulates positive and negative selection, and lineage commitment. $^{8}$ The activation of these various signaling pathways allows thymocytes to integrate information from lymphostromal interactions, which are crucial to determine the fate of $T$ cells within the thymus.

The thymus is divided in two main compartments, cortex and medulla, containing epithelial cells (TEC). T-cell differentiation occurs through TCR-MHC interactions between $T$ cells and TEC and also between T cells and dendritic cells or macrophages embedded within the TEC network. It is noteworthy that mesenchymal cells, such as fibroblasts, are also necessary for T-cell differentiation. ${ }^{9}$ Myoid cells in the thymus correspond to a rare cell population localized in the medulla and at the cortico-medullary junction. ${ }^{10-12}$ They possess the antigenic characteristics of skeletal muscle cells and express MyoD, desmin, troponin $\mathrm{T}$, rapsyn, utrophin and the acetylcholine receptor. ${ }^{11-13}$ Under the term 'myoid cells', defining muscle-like cells, different cells have been described. In testis, myoid cells are involved in the contraction of seminiferous tubules. ${ }^{14}$ The presence of myoid cells in human bone marrow has also been observed during hematopoiesis in embryonic life and in some pathological conditions and autoimmune disorders during adult life. ${ }^{15}$ Thymic myoid cells 
play probably an important role in the thymus as they are well conserved throughout species evolution. However, their biological role remains unclear. Up to now, researchers have mainly been interested in thymic myoid cells because they are suspected to be involved in Myasthenia Gravis: an autoimmune disease characterized by muscle weakness due to autoantibodies directed against acetylcholine receptor and where thymus is the effector site. ${ }^{16,17}$

We are interested in the physiological role of myoid cells within the thymus. We analyzed in vitro thymocyte behavior when cocultured with a myoid cell line developed in our laboratory. ${ }^{11}$ We observed that myoid cells protected thymocytes from apoptosis. We then investigated the biological mechanisms and the signaling pathways involved in this protective effect wondering if it was either due to the activation of prosurvival signaling pathways or to the inhibition of proapoptotic signaling pathways. We also showed that in vitro myoid cells were able to modulate lineage commitment of thymocytes favoring CD4 ${ }^{+} \mathrm{SP}$ thymocytes.

\section{Results}

\section{Myoid immortalized thymic cells (MITC) protected thymocytes from apoptosis in vitro}

To investigate the in vitro effects of myoid cells on thymocytes, freshly isolated thymocytes were cultured in monoculture (control) or in coculture with MITC. The apoptotic state of thymocytes was analyzed at different time points, using flow cytometry.

After $24 \mathrm{~h}$ in coculture with MITC, thymocytes presented a strong decrease of $38.6 \% \pm 3.5(n=13, P<0.0001)$ of the percentage of annexin-V-FITC positive cells in R1 (viable thymocytes) gate (Figure $1 \mathrm{~b}, \mathrm{c}$ ) and an increase of $30.6 \% \pm 5$ ( $n=13, P<0.0001$ ) of the R1/R2 (viable/dead thymocytes) ratio (Figure 1c).

In time course experiments, the decrease in annexin- $V$ FITC labeling due to MITC was detectable from $2 \mathrm{~h}$ after thymocyte seeding, increased progressively up to $24 \mathrm{~h}$ (Figure 1d) and was still observed up to $72 \mathrm{~h}$ (data not shown). We also detected quite rapidly an increase in the $\mathrm{R} 1 / \mathrm{R} 2$ ratio after $6 \mathrm{~h}$ in coculture. These results showed that MITC triggered rapidly a mechanism protecting thymocytes from apoptosis in vitro.

\section{The protective effect of MITC is specific compared to TEC}

To evaluate if this protective role of MITC was specific and not due to a general cellular effect, we compared MITC and TEC effects on thymocyte behavior in vitro. In coculture with MITC, the expected decrease in annexin-V-FITC labeling of thymocytes was observed. In contrast, in coculture with TEC, the annexin-V-FITC labeling of thymocytes was even higher than

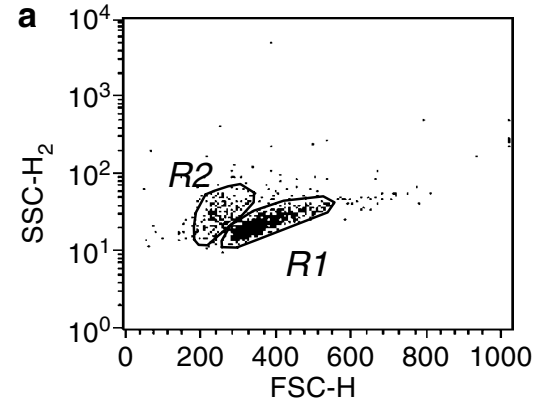

C

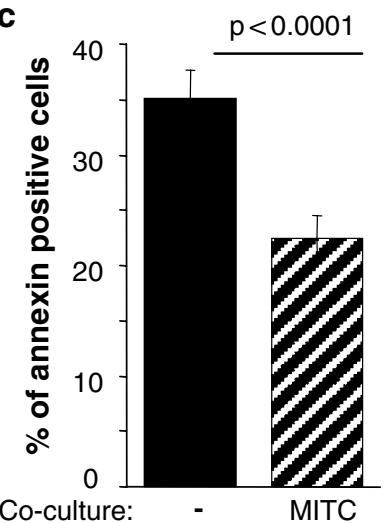

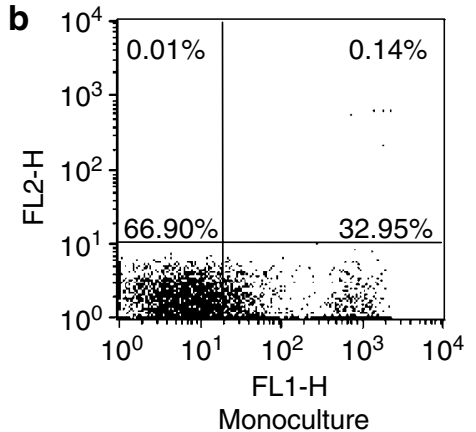
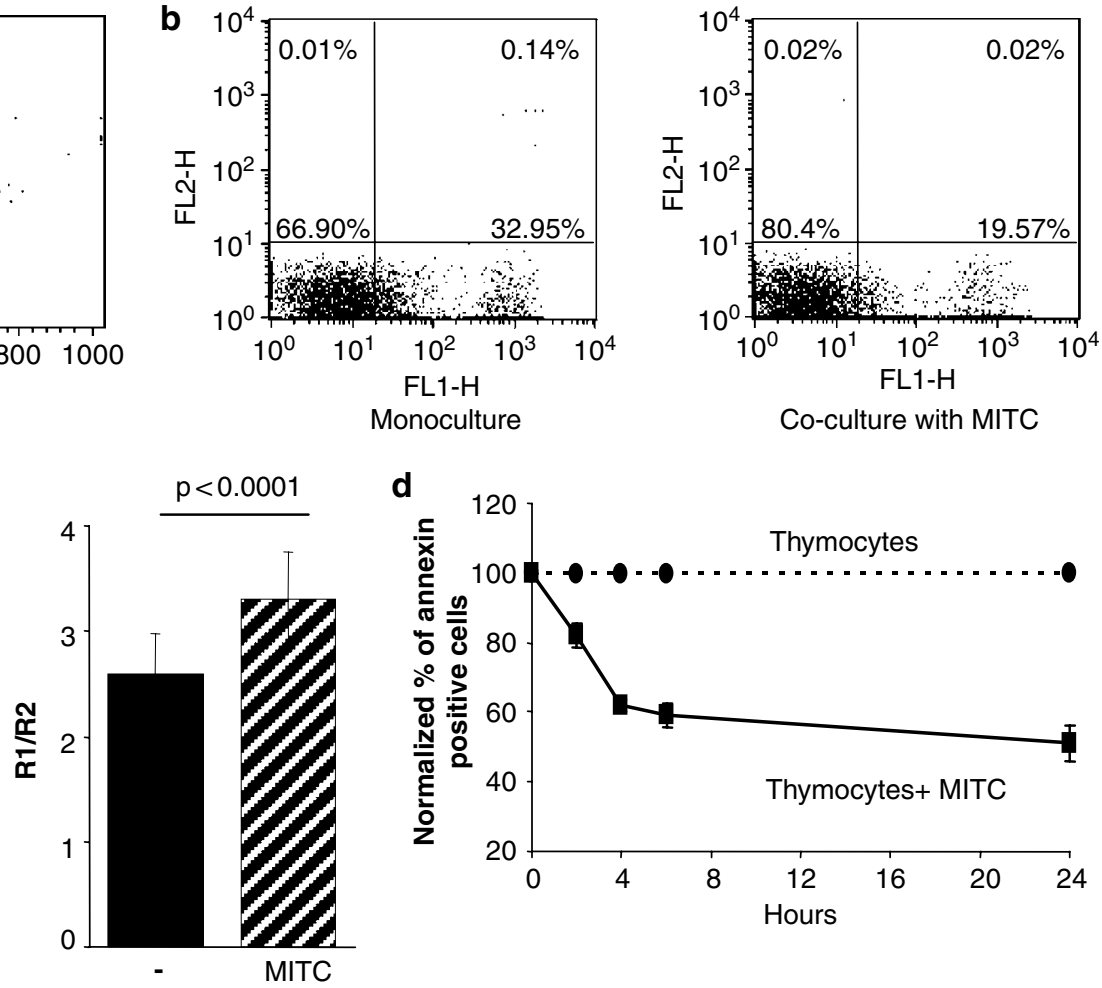

d

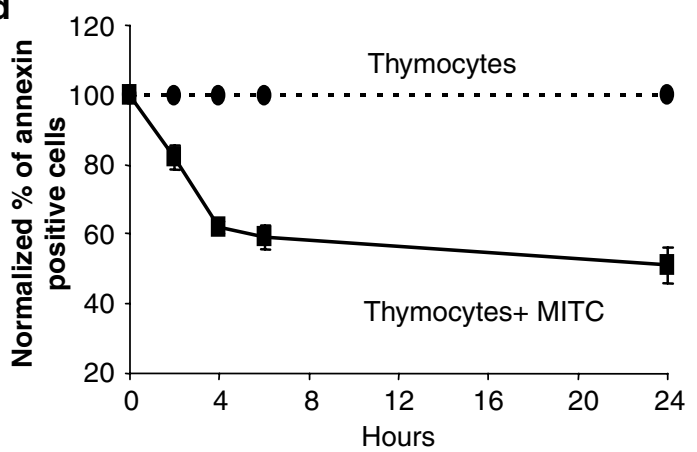

Figure 1 MITC protected thymocytes from apoptosis in vitro. (a) Apoptotic thymocytes present distinct side-scatter (SSC-H) and forward-scatter (FSC-H) features allowing to delimit the R1 (viable thymocytes) and R2 (dead thymocytes) gates. (b) Thymocytes, in monoculture or in coculture with MITC for $24 \mathrm{~h}$, were labeled with annexin-V-FITC (FL1-H) and propidium iodide (FL2-H) and analyzed on a FACScan flow cytometer (representative experiment out of three). (c) Bar charts correspond to the percentage of annexin-V-FITC labeled cells and to the ratio of thymocyte number in R1 gate over R2 gate $(n=13$ experiments $) \pm$ S.E.M. after $24 \mathrm{~h}$ in culture. (d) Time course experiment for annexin-V-FITC labeled thymocytes. For each time point, the data from thymocytes cultured with MITC were compared to those of thymocytes on their own (fixed at 100\%) (representative experiment out of three) 
in monoculture (Figure 2). These data demonstrated that MITC specifically protected thymocytes from apoptosis in vitro compared to TEC.

\section{The protective effect of MITC was dependent on direct cell contacts}

The biological effects observed during coculture could be due to direct cell-to-cell contacts or to the release of soluble factors by myoid cells. ${ }^{11,12,18}$ To avoid any direct cell contacts between MITC and thymocytes, these last were seeded within cell culture inserts. In these culture conditions, we did not observe the decrease in the annexin-V-FITC labeling of thymocytes normally occurring when cultured directly onto MITC. Indeed, the labeling of thymocytes using cell culture inserts was similar for cultures with or without MITC in the lower compartment (Figure $3 a, b)$. We also analyzed the effects of a MITC-conditioned culture medium on the annexinV-FITC labeling of thymocytes after $24 \mathrm{~h}$ in culture (Figure $3 \mathrm{c}$ ). As for experiments using cell culture inserts, the MITCconditioned medium did not protect thymocytes.

These observations demonstrated that the protective effect of MITC on thymocytes was dependent on direct cell-to-cell contacts.

\section{The protective effect of MITC involved Akt and ERK1/2 activation in thymocytes}

We investigated the signaling pathway(s) involved in the protective effect of MITC on thymocytes in vitro. MITC induced Akt and ERK1/2 phosphorylation in thymocytes from $30 \mathrm{~min}$ up to, at least, $24 \mathrm{~h}$ (Figure 4). These MITC-specific phosphorylations were not observed in thymocytes seeded within cell culture inserts demonstrating that cell contacts were necessary as for the MITC protective effect. No activation of JNK/SAPK or p38 MAPK kinases was observed in thymocyte monocultures or when thymocytes were cocultured with MITC. However, both MAPK groups could be activated in our experimental conditions by an ultraviolet $\mathrm{C}$ treatment (data not shown).

We then analyzed if inhibitors of the ERK and Akt pathways could reverse the protective effect of MITC towards thymocytes. Akt is activated downstream of phosphatidylinositol 3-kinase (PI3K) and its activity is inhibited by LY294002 (a PI3K inhibitor). ERK1/2 signaling pathways are inhibited by U0126, an inhibitor of MEK1/2 acting upstream ERKs. ERK1/2 and Akt phosphorylations induced by MITC were efficiently inhibited by U0126 or LY294002 from $2.5 \mu \mathrm{M}$ (Figure 5a, b). Moreover, LY294002 was able to partially inhibit ERK1/2 phosphorylations induced by MITC (Figure 5a). In contrast, Akt phosphorylation was not inhibited in U0126 treated cells (Figure $5 b$ ).

To prove that these kinases were effectively involved in the protective effect of MITC, we investigated the effects of U0126 and LY294002 on the annexin-V-FITC labeling of thymocytes. Cells were treated with U0126 or LY294002 from 5 to $40 \mu \mathrm{M}$. It is worth noting that $\mathrm{U} 0126$ is commonly used at $10 \mu \mathrm{M}$ and LY294002 at $10-100 \mu \mathrm{M}$ to inhibit various cellular functions while they can inhibit the phosphorylation of Akt and ERK1/2 at lower concentrations, respectively. ${ }^{19,20}$ In our experiments, we observed that U0126 and LY294002 inhibited in a dose-dependent manner the protective effect of MITC from 10-20 $\mu \mathrm{M}$ according to the experiment (Figure 5c, d). However, contrary to U0126, LY294002 was not able to
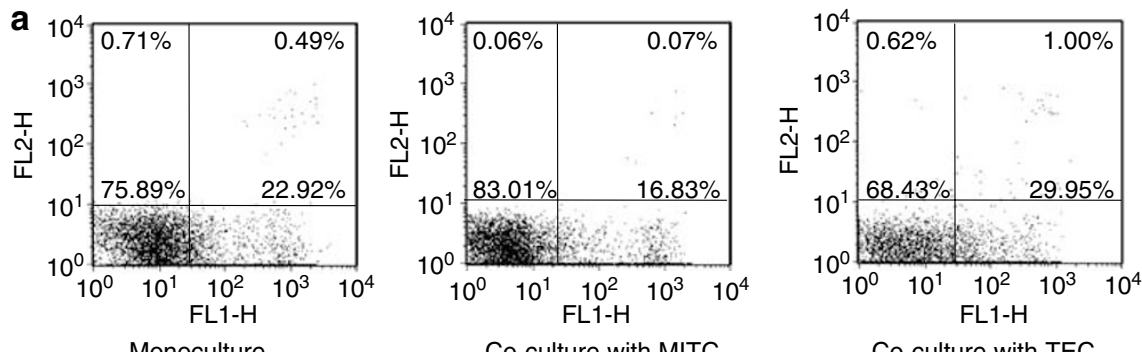

b

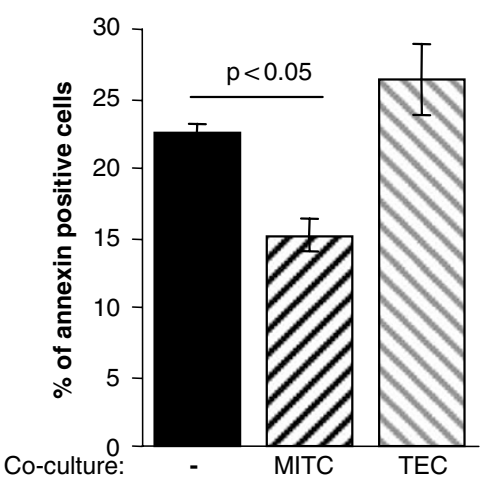

Figure 2 MITC specifically protected thymocytes compared to TEC. Thymocytes in monoculture or in coculture with MITC or TEC for $24 \mathrm{~h}$. (a) Thymocytes were labeled with annexin-V-FITC (FL1-H) and propidium iodide (FL2-H) and analyzed on a FACScan flow (representative experiment out of three). (b) Bar chart corresponds to the percentage of annexin-V-FITC labeled cells (mean value of three experiments \pm S.E.M.) 
a

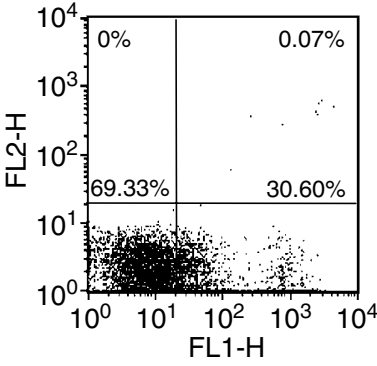

Monoculture

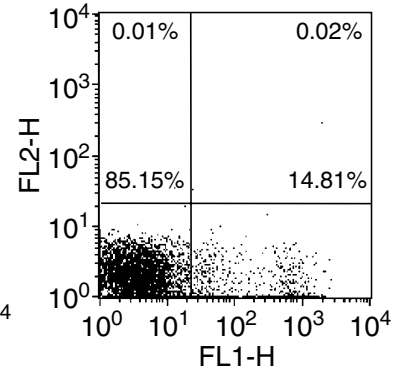

Co-culture with MITC

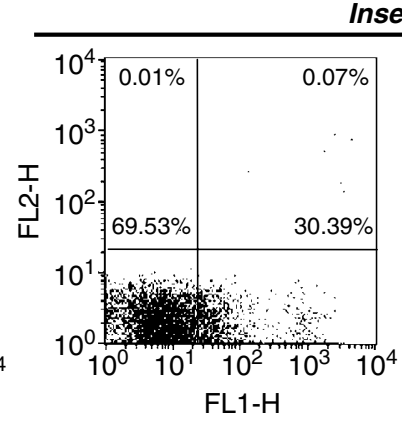

Monoculture
Inserts

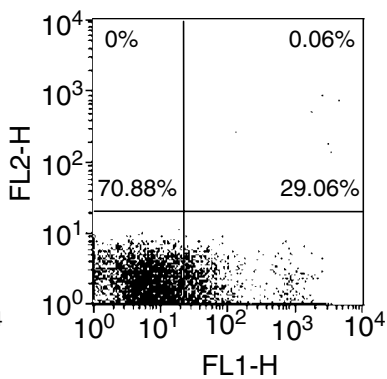

Co-culture with MITC b

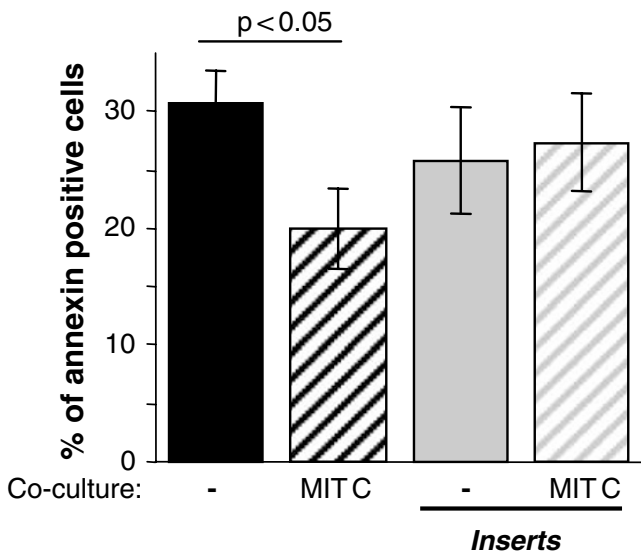

C

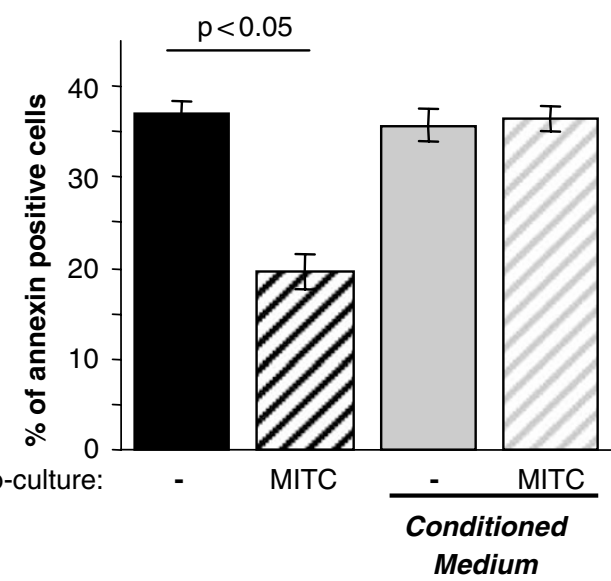

Figure 3 MITC effect on thymocyte survival was dependent on direct cell contacts. Thymocytes in monoculture or in coculture with MITC either directly or within cell culture inserts for $24 \mathrm{~h}$ (representative experiments out of three). (a) Thymocytes were labeled with annexin-V-FITC (FL1-H) and propidium iodide (FL2-H) and analyzed on a FACScan flow. (b) Bar chart corresponds to the percentage of annexin-V-FITC labeled cells (mean value of three experiments \pm S.E.M.). (c) Thymocytes were cultured for $24 \mathrm{~h}$ either in monoculture, in coculture with MITC, with MITC-conditioned medium or with nonconditioned medium (prepared in parallel to MITC-conditioned medium but with no cell). Bar chart corresponds to the percentage of annexin-V-FITC labeled cells (mean value of three experiments \pm S.E.M.)

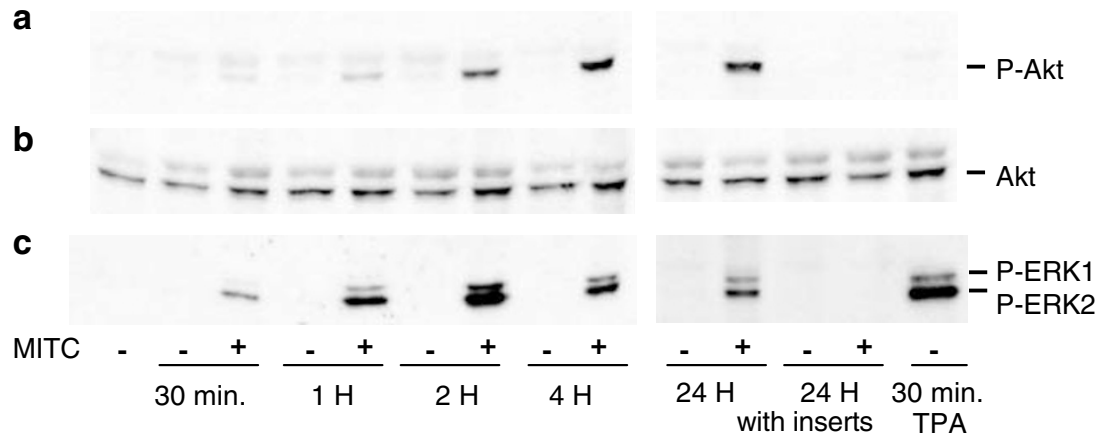

Figure 4 MITC induced Akt and ERK1/2 activation in thymocytes. Thymocytes were cultured for different periods either in monoculture, in direct coculture with MITC or in cell culture inserts with or without MITC in the lower compartment. Thymocytes were also treated with TPA $(100 \mathrm{ng} / \mathrm{ml})$ for 30 min. Proteins were extracted from thymocytes as described in 'Materials and Methods' and cell lysates were immunoblotted with phospho-Akt (a), Akt (b) or phospho-ERK1/2 (c) antibodies; Akt antibody is used as a control of gel loading (representative experiment out of three)

completely block the protective effect of MITC cells towards thymocytes. We carried on the same experiments using a selective inhibitor of Akt (SH-6). We observed from 10 to $20 \mu \mathrm{M}$, an inhibition of the protective effect of MITC towards thymocytes (Figure 5e), confirming the involvement of Akt in the protective effects of MITC. We also wondered if the combined inhibition of Akt and ERK1/2 signaling could be more efficient. However, LY294002 and U0126 used together at $20 \mu \mathrm{M}$, did not lead to a stronger inhibition of the protective effect of MITC compared to when inhibitors were used alone (data not shown).

All these data suggest that the protective effect of MITC was essentially mediated via ERK $1 / 2$ and also partly by Akt; Akt acting probably upstream of ERKs. 
a

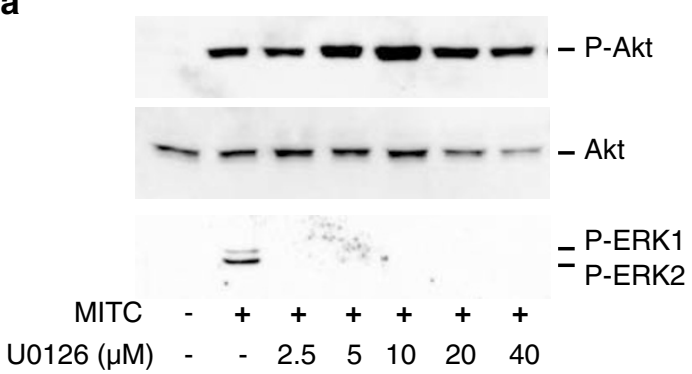

b

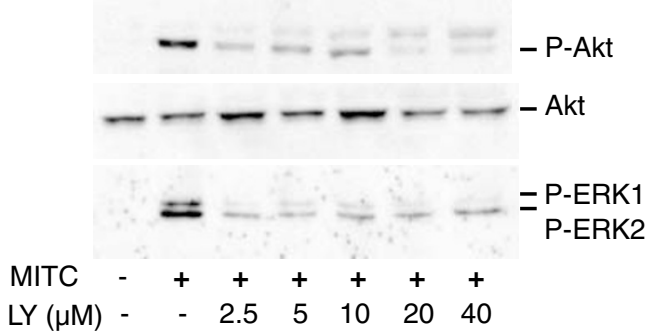

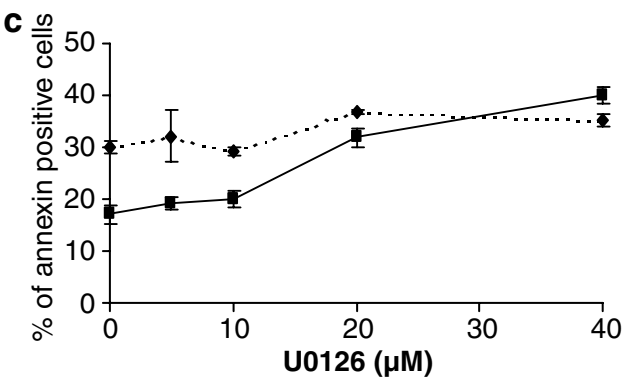

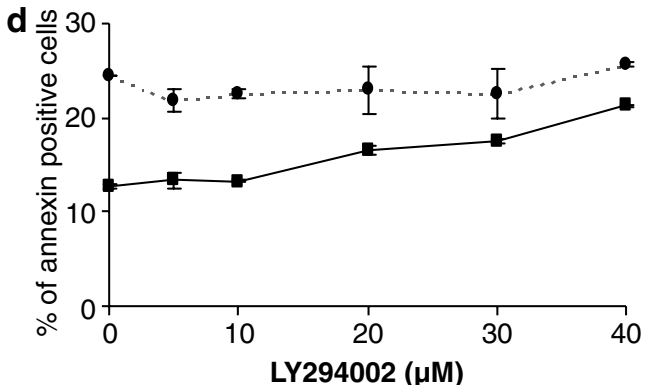

e

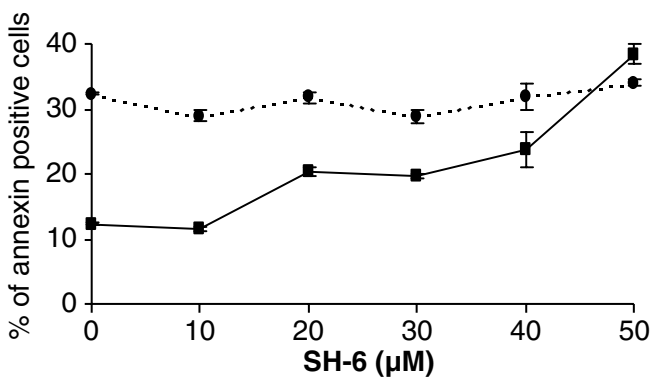

Figure 5 MITC protective effect on thymocytes involved Akt and ERK1/2. Thymocytes were treated with U0126, LY294002 or SH-6 for $24 \mathrm{~h}$ in monoculture or in coculture with MITC (representative experiments out of three). (a, b) Cell lysates, from thymocytes treated with U0126 or LY294002, were immunoblotted with phosphoAkt, Akt and phospho-ERK1/2 antibodies; Akt antibody is used as a control of gel loading. (c-e) Graphs represent the dose-effects of U0126, LY294002 or SH-6 on the annexin-V-FITC labeling of thymocytes in monoculture $(-\bullet-$.$) compared to thymocytes in coculture with MITC (\longrightarrow-)$ for $24 \mathrm{~h}$. The results are expressed as mean values of triplicates \pm S.E.M. (representative experiment out of three)

\section{MITC favored the emergence of SP CD4 ${ }^{+}$over SP CD8 $^{+}$}

From double negative (DN: $\mathrm{CD}^{-} \mathrm{CD}^{-}$) while entering in the thymus, thymocytes become progressively DP and end up SP for either CD4 or CD8. A decrease in $\mathrm{CD}^{+} \mathrm{SP}$ and an increase in $\mathrm{CD} 4{ }^{+} \mathrm{SP}$ were observed when thymocytes were cocultured with MITC (Figure 6a). This difference between the CD4 ${ }^{+}$SP and CD8 ${ }^{+}$SP subpopulations was observed after $24 \mathrm{~h}$ in coculture and was even more important after $48 \mathrm{~h}$ (Figure 6b).

This effect was not due to a differential protective effect of MITC towards particular thymocyte subpopulation. Indeed, using a three-color labeling method (CD4-Cy5, CD8-PE, annexin-V-FITC), we observed a similar protective effect of MITC for CD4 ${ }^{+}$SP or CD8 ${ }^{+}$SP thymocytes (Figure 6c) and also for DP or DN (data not shown).

To determine if, when cocultured with MITC, the difference between the $\mathrm{CD} 4{ }^{+} \mathrm{SP}$ and $\mathrm{CD}{ }^{+} \mathrm{SP}$ subpopulations was not due to an overproliferation to $\mathrm{CD}^{+} \mathrm{SP}$, we used CFSElabeled thymocytes to follow their rate of proliferation. In our culture conditions, thymocytes proliferated at a very low rate with a fluorescence mean varying from $239.5 \pm 2.5$ (duplicate measures) at $24 \mathrm{~h}$ to $130.8 \pm 2.7$ (duplicate measures) at $96 \mathrm{~h}$, in a representative experiment. This was not due to a quenching of the fluorescence as blocking the protein synthesis with cycloheximide blocked the fluorescence mean at $236.7 \pm 8.7(n=8$, duplicate measures made from 24 to $96 \mathrm{~h})$. We cultured CFSE-labeled thymocytes either in monoculture or in coculture with MITC and labeled these cells for CD4 and CD8 at 24, 48 or $72 \mathrm{~h}$. Whatever the culture conditions, we did not observe any significant differences in the fluorescence means for all thymocytes, $\mathrm{CD} 4^{+} \mathrm{SP}$ or $\mathrm{CD}^{+} \mathrm{SP}$ gated in R1 (Figure 6d). Thus, MITC did not decrease the CFSE labeling of CD4 ${ }^{+}$SP subpopulation. They even seemed to slightly slow down the CFSE decrease in all thymocytes subtypes but this was never significant.

Consequently, the decrease of $\mathrm{CD}^{+} \mathrm{SP}$ and the increase in $\mathrm{CD} 4{ }^{+} \mathrm{SP}$ observed when thymocytes were cocultured with MITC, were not due to an increased death of $\mathrm{CD}^{+} \mathrm{SP}$ subpopulation nor to an overproliferation of $\mathrm{CD} 4{ }^{+} \mathrm{SP}$ subpopulation. 


\section{Discussion}

In the experiments described herein, we tried to understand the role of thymic myoid cells and we analyzed their effects on thymocytes. In a first instance, we showed that the human

a

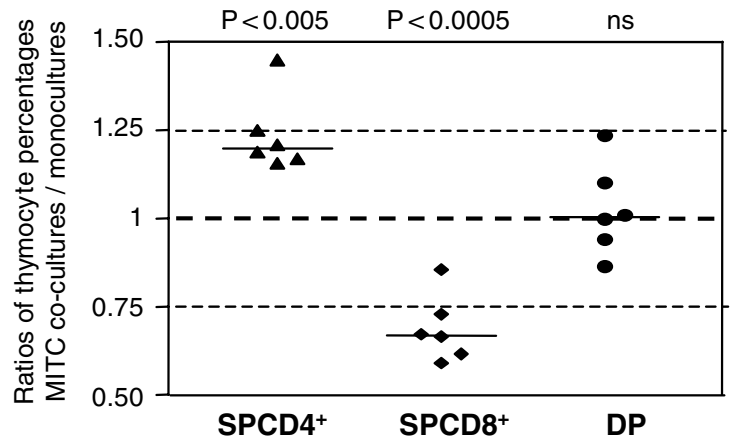

b

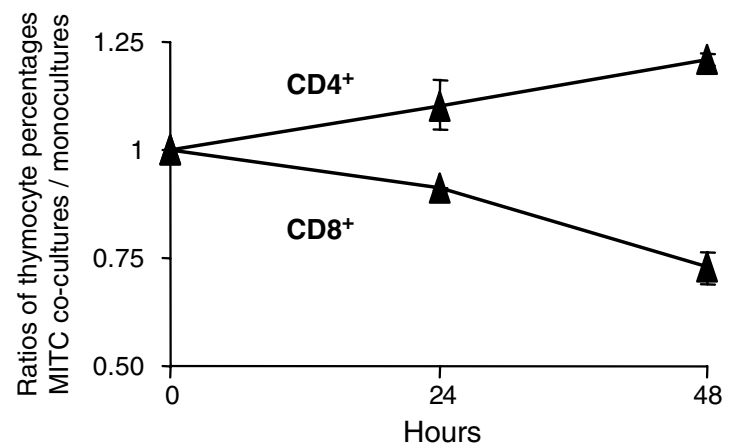

C
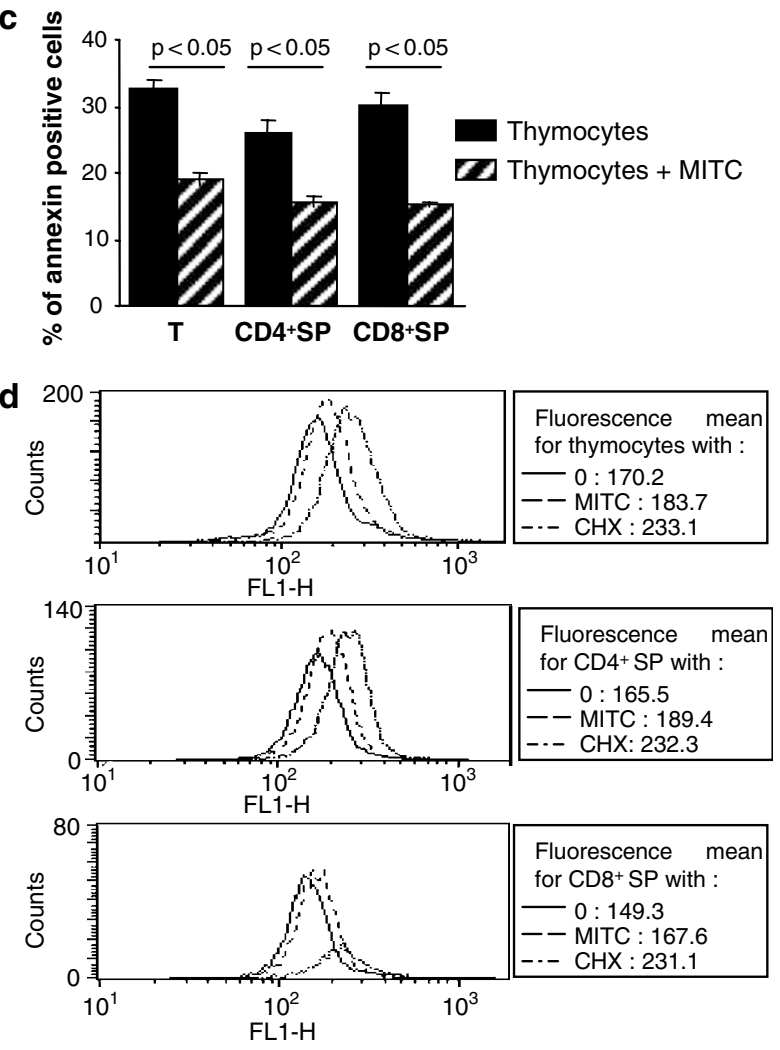

myoid cell line (MITC) protected thymocytes from apoptosis occurring spontaneously in vitro. This was demonstrated by a decrease in the annexin-V-FITC labeling of thymocytes and an increase in the ratio corresponding to the number of viable over dead thymocytes. We then investigated in details this biological phenomenon.

(1) Different observations demonstrated that the protective effect of myoid cells towards thymocytes was not due to an effect on thymocyte proliferation. Indeed, no significant variation in the total number of thymocytes was observed between thymocytes in monoculture or in coculture with MITC (data not shown). We also showed using mitomycin C (an antiproliferative compound) that the protective effect of MITC on thymocytes was similar for mitomycin Cor for nontreated thymocytes. ${ }^{21}$ Moreover, using CFSElabeled thymocytes, we did not detect any significant variation in the means of fluoresecence between thymocytes in monoculture and in coculture with MITC.

(2) We demonstrated that myoid cells protected specifically thymocytes from apoptosis. In our coculture model, using TEC instead of MITC, we increased thymocyte apoptosis in vitro as previously described. ${ }^{22,23}$ It is noteworthy that Rinner et al. analyzed the distinct effects of TEC lines from either the thymus cortex or the medulla. In coculture, both cell lines induced thymocyte apoptosis. However, in coculture with intact fetal thymic fragments, thymocytes were protected from apoptosis induced by medullary TEC but not by cortical TEC. These observations suggest that the thymus microenvironment protected thymocytes from apoptotic signals delivered by medullary TEC. Together with our results, we can hypothesize that myoid cells localized especially in the medulla compartment could counteract apoptotic signals delivered by medullary TEC. ${ }^{24}$ To test this hypothesis, we cocultured thymocytes onto monolayers composed of different proportions of MITC and TEC, especially as TEC possess mainly medullary TEC properties in our culture conditions. ${ }^{11}$ We observed a progressive decrease of the protective effect of MITC as the proportion of TEC increased (data

Figure 6 MITC favored single positive $\mathrm{CD}^{+} \mathrm{SP}$ over single positive $\mathrm{CD}^{+}$SP. Thymocytes, in monoculture or in coculture with MITC, were stained with RPE-Cy5-conjugated anti-CD4 and PE-conjugated anti-CD8 antibodies. (a) For each thymocyte subpopulation, the data correspond to ratios of the percentages of labeled thymocytes in coculture over in monoculture for $24 \mathrm{~h}$. Each point corresponds to the mean value of triplicate measures for six different experiments. $P$ is given for the comparison between thymocytes in monoculture and in coculture for each subpopulation. (b) Analysis of $\mathrm{CD} 4{ }^{+} \mathrm{SP}$ and $\mathrm{CD} 8{ }^{+} \mathrm{SP}$ labeled thymocytes in time course experiment. The data correspond to ratios of the percentages of labeled thymocytes in coculture over in monoculture. Each point corresponds to the mean value of triplicate measures (representative experiment out of three). (c) Bar chart corresponds to the percentage of annexinV-FITC labeled thymocytes after $24 \mathrm{~h}$ in monoculture or in coculture with MITC. The results are expressed as mean values of triplicates \pm S.E.M. for viable thymocytes (R1 gate) and $\mathrm{CD} 4{ }^{+} \mathrm{SP}$ or $\mathrm{CD} 8{ }^{+} \mathrm{SP}$ thymocytes (representative experiment out of three). (d) Thymocytes were labeled with $2 \mu \mathrm{M}$ CFSE and cultured for $72 \mathrm{~h}$ either in coculture with MITC or in monoculture treated or not with $5 \mu \mathrm{g} / \mathrm{ml}$ of cycloheximide (CHX). CFSE labeling was analyzed after a double labeling with RPE-Cy5-CD4 and PE-CD8 antibodies. Histograms represent the CFSE labeling for viable thymocytes and $\mathrm{CD} 4{ }^{+} \mathrm{SP}$ or $\mathrm{CD} 8^{+} \mathrm{SP}$ (fluorescence means are given for the corresponding histograms, representative histograms out of three experiments) 
not shown). These results indicate that both cell types influence thymocyte viability and therefore a high number of myoid cells in the medullary area could affect the viability of the neighboring thymocytes.

(3) We proved that the protective effect of myoid cells towards thymocytes was mediated through direct cell-tocell interactions and was not due to soluble factors. Indeed, MITC were not able to protect thymocytes when they were seeded within cell culture inserts. Moreover, an MITC-conditioned medium did not affect the spontaneous apoptosis of thymocytes in vitro. However, myoid cells secrete soluble factors ${ }^{11,12,18}$ and Kamo et al. observed that conditioned culture medium from rat thymic myoid cells can stimulate thymocyte proliferation. ${ }^{25}$ What kind of direct cellular interactions trigger the protective effect of myoid cells ? Numerous membrane components could be involved and one could wonder whether $\mathrm{MHC}$ molecules are implicated. Our data do not support this hypothesis. (i) MITC does not express MHC class II molecules; ${ }^{11,12}$ (ii) MITC express MHC class I molecules but TEC also express almost exclusively MHC class I molecules in our culture conditions ${ }^{26}$ while they did not protect thymocytes from apoptosis and even induced it. Therefore, the implication of TCR-MHC interactions in the protective effect of myoid cells towards thymocytes can be excluded. We previously demonstrated the expression on myoid cells of LFA-3 (CD58) antigen; which could allow thymocyte-myoid cell interactions. ${ }^{12}$ However, as for MHC class I molecules, LFA-3 is also expressed by TEC and the molecule(s) involved in the protective effect of myoid cells is (are) probably specifically expressed on these cells and not on TEC.

The protective effect of myoid cells on thymocytes was triggered rapidly from $2 \mathrm{~h}$ in coculture, suggesting that myoid cells modulated early signaling pathway(s) essential for thymocyte protection.

We wondered if it was due to the activation of prosurvival signaling pathways involving Akt or ERKs, or if it was due to the inhibition of proapoptotic signaling pathways as those involving JNK/SAPK or p38 MAPK. Indeed, apoptosis has been directly linked to JNK/SAPK or p38 MAPK activation. ${ }^{27}$ However, we were not able to detect any activation of JNK/ SAPK and p38 MAPK in thymocytes cultured with or without MITC (data not shown). In contrast, MITC induced an early and prolonged activation ERK1/2 and Akt in thymocytes. We then demonstrated that ERK1/2 and Akt activation in thymocytes were effectively involved in the protective effect triggered by MITC. First, ERK1/2 and Akt activations were dependent on direct MITC-thymocyte contacts as was the decrease of annexin-V-FITC labeling of thymocytes. Secondly, inhibitors of ERK1/2 (U0126) and Akt (LY294002 or $\mathrm{SH}-6)$ signaling pathways inhibited the protective effect of MITC towards thymocytes. We also underlined that Akt and ERK1/2 might be involved in a single signaling pathway. Indeed, when used together U0126 and LY294002 did not have more effect in inhibiting the MITC protective effect. Moreover, LY294002 partially inhibited ERK1/2 phosphorylations induced by MITC, while U0126 did not inhibit Akt phosphorylation, suggesting that ERK1/2 were activated downstream of the PI3K/Akt pathway. However, as Akt inhibition seems to be less efficient than ERK1/2 inhibition to cancel the protective effect of myoid cells towards thymocytes, we suggest that other kinases could act upstream ERK1/2, typically MAPK-ERK kinase (MEK) 1/2. Although the ERK1/2 and Akt signaling pathways are usually described as independent, recent reports suggest a certain degree of crosstalk between these two cascades. Na et al. ${ }^{28}$ described that Akt enhanced ERK activity modulating thymocyte selection and T-cell activation. The involvement of Akt in cell survival is well documented. ${ }^{2,6}$ Concerning ERKs, their prosurvival effects have been mainly linked to their ability to stimulate cell proliferation. However, as for Akt, ERKs can also directly influence cell survival. Akt and ERKs induce the expression of antiapoptotic molecules such as Bcl-2 and $\mathrm{Bcl}-\mathrm{xl},{ }^{29,30}$ but they also inhibit the expression of pro-apoptotic molecules or their activity. For example, Akt and ERKs phosphorylate Bad on distinct serines blocking its proapoptotic effects. ${ }^{31,32}$ They also phosphorylate and inhibit caspase-9 promoting cell survival. ${ }^{33,34}$

To conclude about the protective effect of MITC towards thymocytes, we suggested that MITC activated in thymocytes, a 'prosurvival' or 'antiapoptotic' signaling pathway involving especially ERK1/2 but also Akt which is acting upstream of ERK1/2.

Thymocyte behavior is tightly linked to interactions with stromal thymic cells. In the process of understanding the role of myoid cells, we also analyzed their effects on different thymocyte subpopulations. We observed a progressive increase in $\mathrm{CD}^{+}{ }^{+} \mathrm{SP}$ and a decrease in $\mathrm{CD} 8^{+} \mathrm{SP}$ populations when thymocytes were cocultured with MITC. These variations in the $\mathrm{CD} 4{ }^{+} \mathrm{SP}$ and CD8 ${ }^{+} \mathrm{SP}$ could not be explained by a differential protective effect of MITC towards distinct thymocyte subpopulation as MITC equally decreased the annexin-V-FITC labeling of $\mathrm{CD}^{+}{ }^{+} \mathrm{SP}, \mathrm{CD} 8^{+} \mathrm{SP}, \mathrm{DP}$ or $\mathrm{DN}$ thymocytes.

We can hypothesize that MITC favored CD4 ${ }^{+} \mathrm{SP}$ versus $\mathrm{CD}^{+} \mathrm{SP}$ proliferation. However, if the increase in $\mathrm{CD} 4{ }^{+} \mathrm{SP}$ thymocytes was due to a specific overproliferation of this subpopulation, we should have globally observed a decrease in the number of $\mathrm{CD}^{+} \mathrm{SP}$ but also of DP and DN. We did observe a decrease in $\mathrm{CD} 8{ }^{+} \mathrm{SP}$ but no concordant decrease in DP and DN. Moreover, using CFSE-labeled thymocytes, we did not detect any significant differences in the proliferative state of $\mathrm{CD} 4{ }^{+} \mathrm{SP}$ in monoculture or in coculture with MITC. It seems thus that the observed variations in the CD4 ${ }^{+} \mathrm{SP}$ and $\mathrm{CD}^{+}$SP subpopulations is due to something more complex than a simple over-proliferation of $\mathrm{CD} 4^{+} \mathrm{SP}$ thymocytes when cocultured with myoid cells.

We can also consider that the $\mathrm{CD} 4{ }^{+} \mathrm{SP}$ increase and the $\mathrm{CD}^{+} \mathrm{SP}$ decrease are due to an effect of MITC on thymocyte differentiation. The differentiation of DP thymocytes in either $\mathrm{CD} 4{ }^{+} \mathrm{SP}$ or $\mathrm{CD}^{+} \mathrm{SP}$ (lineage commitment) has been essentially linked to TCR-MHC interactions: DP thymocytes expressing $\mathrm{MHC}$ class II specific TCR differentiate into $\mathrm{CD}^{+}{ }^{+} \mathrm{SP}$ whereas those expressing $\mathrm{MHC}$ class I specific TCR differentiate into $\mathrm{CD}^{+} \mathrm{SP}$. MITC express $\mathrm{MHC}$ class I but no $\mathrm{MHC}$ class II molecules. ${ }^{11,12}$ Consequently, if the lineage commitment was strictly determined by the specificity of the TCR-MHC interactions, we should have ended up with 
more $\mathrm{CD} 8{ }^{+} \mathrm{SP}$ than $\mathrm{CD} 4{ }^{+} \mathrm{SP}$ thymocytes in our coculture model with MITC as they only express only MHC class I molecules. ${ }^{11,12}$ Consequently, thymocyte differentiation do not seem to only depend on the specificity of the TCR-MHC interactions.

The protein tyrosine kinase Lck, which is expressed mainly in T cells throughout development, associates noncovalently with the cytoplasmic tails of both CD4 and CD8. Lck associates more efficiently with CD4 than CD8 and the level of total Lck activation seems to determine CD4 differentiation (strong Lck activation) or CD8 differentiation (weak Lck activation). However, it has been shown that thymocytes carrying a class I restricted TCR developed into functional CD4 T cells when Lck activity was increased. ${ }^{35}$ Moreover, in experiments where TCR were crosslinked with conventional antibodies, CD4 but not CD8 thymocytes were generated presumably because these antibodies aggregated and gave a strong signal activating Lck. ${ }^{36}$ We can thus hypothesize that the MHC class I signal delivered exclusively by MITC in our coculture model overwhelmed normal TCR-MHC class I interaction, strongly activating Lck and favoring $\mathrm{CD} 4{ }^{+} \mathrm{SP}$ differentiation instead of $\mathrm{CD}^{+} \mathrm{SP}$.

Lineage commitment has also been shown to be dependent on the activation of the ERK and Akt pathways: ERK and Akt activation both favored positive selection toward the CD4 lineage. ${ }^{28,37}$ In coculture with MITC, we observed a prolonged activation of Akt and ERK1/2, which could thus favor DP thymocyte differentiation in $\mathrm{CD} 4{ }^{+} \mathrm{SP}$ instead of $\mathrm{CD}^{+} \mathrm{SP}$ thymocytes. These observations suggest that MITC could modulate thymocyte differentiation in vitro. Thymic myoid cells, localized at the cortico-medullary junction and in the medulla, could deliver additional signals necessary for a complete thymocyte differentiation. Similarly, myoid cells from the bone marrow have been involved in the regulation of hematopoietic precursor behavior. ${ }^{38}$ Our data, together with the literature, underline that T-cell maturation in the thymus is under the control of the thymic microenvironment. If the thymic epithelium (cortical and medullary TEC) is known to play an important role in T-cell development, other thymic stromal cells seem to be indispensable. Mesenchyme-derived fibroblasts are required for early T-cell development. ${ }^{9}$ Dendritic cells and macrophages are involved in the proper thymocyte differentiation and even in thymocyte survival. ${ }^{39-41}$ The end of the differentiation process for thymocytes occurs in the medulla where they have been shown to reside up to 2 weeks before being exported to the periphery. What happens during this period is not well known. Myoid cells are well conserved throughout species evolution and are probably indispensable for the maturation process of thymocytes. Myoid cells could protect thymocytes from the apoptotic signals delivered by other stromal cells but could also provide additional signals necessary for thymocytes to complete their differentiation.

\section{Materials and Methods}

\section{Cells}

Myoid cell line (MITC) was established in our laboratory as described in Wakkach et al. ${ }^{11}$ Briefly, thymic stromal cells were immortalized using a plasmid vector encoding the SV40T oncogene. Among the cell lines obtained, one possessed cellular characteristics and markers of skeletal muscle cells observed on normal myoid cells. In all experiments, we used an RPMI medium composed of RPMI 1640 with Glutamax I supplemented with $10 \%$ fetal calf serum, unless otherwise stated.

Thymic epithelial cells (TEC) were cultured from fresh thymic fragments finely cut with scissors and seeded onto cell culture flasks as previously described. ${ }^{25}$ The culture medium for TEC was composed of RPMI 1640 with Glutamax I supplemented with $20 \%$ horse serum and $0.2 \%$ ultroser $\mathrm{G}$. This medium was changed every $2-3$ days for 14 days. TEC were recovered after trypsin digestion, filtered through a gaze, centrifuged $10 \mathrm{~min}$ at $1200 \mathrm{rpm}$ and directly seeded for coculture experiments.

Thymocytes were obtained from fresh thymic fragments. Thymic fragments were cleaned and cut with scissors in HBSS, releasing thymocytes in the medium. Thymocytes were recovered after filtration through a gaze, centrifuged $10 \mathrm{~min}$ at $1200 \mathrm{rpm}$ and homogenized in RPMI medium.

In all experiments, we used fresh thymic fragments from children undergoing corrective cardiovascular surgery at the Marie Lannelongue hospital (Le Plessis Robinson, France). All cell culture products were from Gibco Life Technologies (Cergy-Pontoise, France).

\section{Cell culture models}

MITC and TEC possess different proliferation properties. For our cocultures, these cells were thus seeded at different rates (respectively, $2 \times 10^{4}$ or $1 \times 10^{5}$ cells/well in 24-well plates (Becton Dickinson-Falcon, Le pont de claix, France)) in order to reach sub-confluency after 3 days (around $2.5 \times 10^{5}$ cells/well). Freshly isolated thymocytes were then seeded in RPMI medium at $1 \times 10^{6}$ cells/well onto 3-day-old MITC cultures, TEC cultures or directly in empty wells. Thymocytes were recovered from these cocultures at different time points according to the experiments. A small percentage of thymocytes bound to MITC but these interactions were quite easily disrupted.

This coculture model was also modified and thymocytes were cultured within cell culture inserts for 24-well plates $(1 \mu \mathrm{m}$ pore size, Becton Dickinson-Falcon). Thymocytes and MITC were separated in two different compartments delimitated by the microporous membrane of the inserts, which prevents cell migration through pores but allows the diffusion of most soluble molecules.

MITC-conditioned culture medium was prepared by incubating MITC for three days in RPMI medium. A nonconditioned culture medium was prepared in parallel to MITC-conditioned medium in plates containing no cell. After 3 days in the incubator, supernatants were collected, centrifuged and used onto freshly isolated thymocytes for $24 \mathrm{~h}$ in culture.

To analyze the effects of pharmacological compounds, thymocytes were treated just after seeding. LY294002 (PI3-kinase inhibitor) and U0126 (MAPK/ERK kinase 1/2 (MEK1/2) inhibitor) were from Alexis Biochemicals (Qbiogene, llkirch, France) and SH-6 (Akt inhibitor) was from Calbiochem (VWR, Fontenay-sous-Bois, France).

\section{Evaluation of the apoptotic state of thymocytes using FACS flow cytometry}

Apoptosis was evaluated by quantifying phosphatidylserine residues exposed on apoptotic cell membranes. For each assay, $1 \times 10^{6}$ thymocytes were washed with PBS and incubated in $100 \mu \mathrm{l}$ of binding buffer (10 mM HEPES/NaOH pH 7.4, $140 \mathrm{mM} \mathrm{NaCl,} 5 \mathrm{mM} \mathrm{CaCl}$ ) containing $1 \mu \mathrm{l}$ of human recombinant FITC-conjugated annexin-V (Roche 
Diagnostics, Meylan, France) and $2 \mu \mathrm{l}$ of propidium iodide $(50 \mu \mathrm{g} / \mathrm{ml}$, Becton Dickinson). After 15 min of incubation in the dark, a dual color analysis was performed on a FACScan flow cytometer (Becton Dickinson). In the analysis conditions used, the side-scatter (SSC-H) and forwardscatter (FSC-H) features have been determined to gate precisely on thymocytes. MITC correspond to bigger cells compared to thymocytes and even if a few myoid cells detached themselves from the plate they could not interfere with the measures on thymocytes. Apoptotic thymocytes present distinct SSC and FSC features allowing to delimitate the R1 and $\mathrm{R} 2$ gates (Figure 1a): the $\mathrm{R} 1$ gate contains viable cells while the $\mathrm{R} 2$ gate contains mainly apoptotic cells (with $95.6 \% \pm 1.6(n=5)$ annexin and $\mathrm{PI}$ positive cells). The ratio of the number of thymocytes in R1 over R2 was evaluated. The percentage of annexin-V-FITC stained thymocytes was analyzed in the R1 gate on a log FL1 versus log FL2 dot plot where quadrants were adjusted according to unstained control cells.

\section{Analysis of the behavior of thymocyte subpopulations using FACS flow cytometry}

To analyze thymocyte subpopulations $\left(\mathrm{CD} 4^{+} \mathrm{SP}, \mathrm{CD} 8^{+} \mathrm{SP}, \mathrm{DP}, \mathrm{DN}\right)$ thymocytes were stained with R-phycoerythrin (RPE)-Cy5-conjugated anti-CD4 (Dako, Trappes, France) and phycoerythrin (PE)-conjugated anti-CD8 (Dako) antibodies for $30 \mathrm{~min}$ at $4^{\circ} \mathrm{C}$ before being labeled with annexin-V-FITC. The analysis was performed on a FACScan flow cytometer and the proportion of annexin-V-FITC positive cells was analyzed in $\mathrm{CD} 4^{+} \mathrm{SP}, \mathrm{CD} 8^{+} \mathrm{SP}, \mathrm{DP}$ and $\mathrm{DN}$ thymocyte subpopulations.

Cellular proliferation of thymocyte subpopulations was analyzed by labeling thymocytes with CFSE (Carboxyfluorescein diacetate succinimidyl ester, Molecular Probes/Invitrogen, Cergy-Pontoise, France). CFSE spontaneously and irreversibly couples to cellular proteins and a proportional decrease in CFSE labeling can thus be observed as cells divide. Freshly isolated thymocytes were washed twice in PBS and $1 \times 10^{7} \mathrm{cells} / \mathrm{ml}$ were labeled with $2 \mu \mathrm{M}$ CFSE for $10 \mathrm{~min}$ at $37^{\circ} \mathrm{C}$ in the dark. The staining was terminated by two washes in RPMI medium supplemented with $10 \%$ fetal calf serum and labeled thymocytes were seeded as previously described. Labeled thymocytes were also treated with $5 \mu \mathrm{g} / \mathrm{ml}$ of cycloheximide (Sigma, Saint Quentin Fallavier, France) to block protein synthesis, inhibiting consequently the CFSE decrease (negative control). After different times, thymocytes were stained with RPE-Cy5-conjugated anti-CD4 and PE-conjugated anti-CD8 antibodies and analyzed on a FACScan flow cytometer on the R1 (viable thymocytes) gate and on either $\mathrm{CD} 4{ }^{+} \mathrm{SP}$ or $\mathrm{CD} 8{ }^{+} \mathrm{SP}$ thymocyte gates.

\section{Western blot analysis}

Thymocytes were recovered in ice-cold PBS, centrifuged at $1200 \mathrm{rpm}$ for $5 \mathrm{~min}$ at $4^{\circ} \mathrm{C}$ and the pellets resuspended in lysis buffer containing $25 \mathrm{mM}$ HEPES (pH 8), $0.2 \mathrm{mM}$ EDTA, $1.5 \mathrm{mM} \mathrm{MgCl} 2,0.3 \mathrm{M} \mathrm{NaCl}, 0.1 \%$ Triton $X$ $100,0.1 \mathrm{mM}$ orthovanadate, $2 \mathrm{mM}$ PMSF and protease inhibitors (Complete, Roche Diagnostics). Cell suspensions were rotated at $4^{\circ} \mathrm{C}$ for $30 \mathrm{~min}$ and the extracts cleared by centrifugation at $13000 \times \mathrm{g}$ for $10 \mathrm{~min}$ at $4^{\circ} \mathrm{C}$. The supernatants were mixed with $4 \times$ SDS-PAGE sample buffer. Supernatant fractions containing equal amounts of protein were then separated by $10 \%$ SDS-polyacrylamide gels and transferred onto polyvinylidene difluoride membrane (PerkinElmer Life Sciences, Courtaboeuf, France).

The membranes were blocked for $1 \mathrm{~h}$ with $5 \%$ dried milk in TBST $(20 \mathrm{mM}$ Tris- $\mathrm{HCl}$ (pH 8), $150 \mathrm{mM} \mathrm{NaCl}$ and $0.05 \%$ Tween-20) and incubated overnight at $4{ }^{\circ} \mathrm{C}$ in TBST containing $5 \%$ BSA with antibodies recognizing either phospho-Akt, Akt or phospho-ERK1/2 (1:1000; Cell Signaling - Ozyme, Saint Quentin en Yveline, France). After 3-4 washes of 5-10 min in TBST, the membranes were incubated for $1 \mathrm{~h}$ with a donkey peroxidase-conjugated anti-rabbit IgG antibody (1:2000; Amersham Biosciences, Orsay, France) in TBST containing 5\% dried milk. The membranes were finally washed 3-4 times $5-10 \mathrm{~min}$ in TBST before the detection of the immune signal using the ECL method (Lumi Light ${ }^{\text {plus }}$, Roche Diagnostics).

\section{Statistical analysis}

Results are expressed as mean \pm S.E.M. The paired Student's $t$-test was used throughout and $P$ was always calculated compared to thymocyte monocultures.

\section{Acknowledgements}

We thank Sylvia Cohen-Kaminsky for helpful discussions. This work was supported by the National Institute of Health Grant NS 39869, the European Community Grant QLG1-CT 2001-01918, the 'Universite Paris-Sud', the 'Centre National de la Recherche Scientifique (CNRS)' and the 'Association Française contre les myopathies (AFM)'.

\section{References}

1. Germain R (2002) T-cell development and the CD4-CD8 lineage decision. Nat. Rev. Immunol. 2: 309-322

2. Cantrell D (2002) Protein kinase $B$ (Akt) regulation and function in $T$ lymphocytes. Immunology 14: 19-26

3. Bauer $B$ and Baier $G$ (2002) Protein kinase $C$ and AKT/protein kinase $B$ in CD4+ T-lymphocytes: new partners in TCR/CD28 signal integration. Mol. Immunol. 38: 1087-1099

4. Hibi M, Lin A, Smeal T, Minden A and Karin M (1993) Identification of an oncoprotein- and UV-responsive protein kinase that binds and potentiattes the c-Jun activation domain. Genes Dev. 7: 2135-2148

5. Rousse J, Cohen P, Trigon S, Morange M, Alonso-Llamazares A, Zamanillo D, Hunt T and Nebreda AR (1994) A novel kinase cascade triggered by stress and heat shock that stimulates MAPKAP kinase- 2 and phosphorylation of the small heat shock proteins. Cell 78: 1027-1037

6. Cross T, Scheel-Toellner D, Henriquez N, Deacon E, Salmon M and Lord J (2000) Serine/threonine protein kinases and apoptosis. Exp. Cell Res. 256:3441

7. Hoshi M, Nishida E and Sakai H (1988) Activation of a Ca2+-inhibitable protein kinase that phosphorylates microtubule-associated protein 2 in vitro by growth factors, phorbol esters, and serum in quiescent cultured human fibroblasts. J. Biol. Chem. 263: 5396-5401

8. Rincon M (2001) MAP-kinase signaling pathways in T cells. Curr. Opin. Immunol. 13: 339-345

9. Suniara RK, Jenkinson EJ and Owen JJ (2000) An essential role for thymic mesenchyme in early T cell development. J. Exp. Med. 191: 1051-1056

10. Drenckhahn D, von Gaudecker B, Muller-Hermelink $H$, Unsicker $K$ and Groschel-Stewart U (1979) Myosin and actin containing cells in the human postnatal thymus. Ultrastructural and immunohistochemical findings in normal thymus and in myasthenia gravis. Virchows Arch. B. Cell Pathol. Incl. Mol. Pathol. 23: 33-45

11. Wakkach A, Poea S, Chastre E, Gespach C, Lecerf F, De La Porte S, Tzartos S, Coulombe A and Berrih-Aknin S (1999) Establishment of a human thymic myoid cell line. Phenotypic and functional characteristics. Am. J. Pathol. 155: 1229-1240

12. Wakkach A, Chastre E, Bruand C, Cohen-Kaminski S, Emami S, Gespach C and Berrih-Aknin S (2001) Phenotypic and functional characterization of human thymic stromal cell lines. Cell. Mol. Biol. 47: 167-178 
13. Mesnard-Rouiller L, Bismuth J, Wakkach A, Poea-Guyon S and Berrih-Aknin S (2004) Thymic myoid cells express high levels of muscle genes. J. Neuroimmunol. 148: 97-105

14. Rossi F, Ferraresi A, Romagni $P$, Silvestroni $L$ and Santiemma V (2002) Angiotensin II stimulates contraction and growth of testicular peritubular myoid cells in vitro. Endocrinology 143: 3096-3104

15. Papadopoulos N, Simopoulos C, Kotini A, Lambropoulou M, Tolparidou I and Tamiolakis D (2001) Differential expression of alpha-smooth muscle actin molecule in a subset of bone marrow stromal cells, in b-cell chronic lymphocytic leukemia, autoimmune disorders and normal fetuses. Eur. J. Gynaecol. Oncol. 22: $447-450$

16. Roxanis I, Micklem K, McConville J, Newsom-Davis J and Willcox N (2002) Thymic myoid cells and germinal center formation in myasthenia gravis; possible roles in pathogenesis. J. Neuroimmunol. 125: 185-197

17. Matsumoto MY, Matsuo H, Oka T, Fukudome T, Hayashi K, Shiraishi $H$, Motomura M, Shibuya N and Ayabe $\mathrm{H}$ (2004) Thymic myoid cells as a myasthenogenic antigen and antigen-presenting cells. J. Neuroimmunol. 150: 80-87

18. Iwakami N, Kikuchi A, Kunishita T, Yamamoto H, Nonaka I and Kamo I (1996) Analysis of lymphoproliferative cytokines produced by thymic myoid cells. Immunology 87: 108-112

19. Favata MF, Horiuchi KY, Manos EJ, Daulerio AJ, Stradley DA, Feeser WS, Van Dyk DE, Pitts WJ, Earl RA, Hobbs F, Copeland RA, Magolda RL, Scherle PA and Trzaskos JM (1998) Identification of a novel inhibitor of mitogen-activated protein kinase kinase. J. Biol. Chem. 273: 18623-18632

20. Adi S, Wu NY and Rosenthal SM (2001) Growth factor-stimulated phosphorylation of Akt and p70S6K is differentially inhibited by LY294002 and wortmannin. Endocrinology 142: 498-501

21. Le Panse-Ruskoné R and Berrih-Aknin S (2003) Human myoid cells protect thymocytes from apoptosis. Ann. NY Acad. Sci. 998: 266-270

22. Popovic P, Colic M, Vucevic D, Gasic S and Pavicic $L$ (2000) Comparison of signaling pathways involved in apoptosis of a thymocyte hybridoma triggered by a rat thymic medullary epithelial cell line, dexamethasone or T-cell receptor cross-linking. Immunol. Lett. 72: 83-91

23. Sharova NI, Dzutsev AK, Litvina MM, Pleskovskaya GN, Kharchenko TY and Yarilin AA (2001) Thymic epithelial cells induce Fas-independent activation apoptosis of thymocytes. Immunol. Lett. 78: 201-207

24. Rinner I, Eren R, Skreiner E, Kukulansky T, Kasai M, Hirokawa K, Globerson A and Schauenstein K (1996) Thymocyte-directed enhancement of apoptosis via soluble factor(s) derived from a cortical and a medullary thymic epithelial cell line. Cell Tissue Res. 284: 327-330

25. Kamo I, Tada-Kikuchi A, Furukawa S, Iwasaki Y, Nonaka I, Satoyoshi E and Itoh T (1985) Effects of thymic myoid cell culture supernatant on cells from lymphatic tissues. Cell immunol. 94: 587-597

26. Berrih S, Arenzana-Seisdedos F, Cohen S, Devos R, Charron D and Virelizier $\mathrm{JL}$ (1985) Interferon-gamma modulates HLA class II antigen expression on cultured human thymic epithelial cells. J. Immunol. 135: 1165-1171
27. Tobiume K, Matsuzawa A, Takahashi T, Nishitoh H, Morita K, Takeda K, Minowa O, Miyazono K, Noda T and Ichijo H (2001) ASK1 is required for sustained activations of JNK/p38 MAP kinases and apoptosis. EMBO Rep. 2: 222-228

28. Na S, Patra A, Scheuring Y, Marx A, Tolaini M, Kioussis D, Hemmings B, Hunig $T$ and Bommhardt U (2003) Constitutively active protein kinase B enhances Lck and Erk activities and influences thymocyte selection and activation. $\mathrm{J}$ Immunol. 171: 1285-1296

29. Pugazhenthi S, Nesterova A, Sable C, Heidenreich KA, Boxer LM, Heasley LE and Reusch JE (2000) Akt/protein kinase B up-regulates Bcl-2 expression through cAMP-response element-binding protein. J. Biol. Chem. 275: 10761-10766

30. Pardo O, Arcaro A, Salerno G, Raguz S, Downward J and Seckl M (2002) Fibroblast growth factor-2 induces translational regulation of $\mathrm{Bcl}-\mathrm{XL}$ and $\mathrm{Bcl}-2$ via a MEK-dependent pathway: correlation with resistance to etoposideinduced apoptosis. J. Biol. Chem. 277: 12040-12046

31. del Peso L, Gonzalez-Garcia M, Page C, Herrera R and Nunez G (1997) Interleukin-3-induced phosphorylation of BAD through the protein kinase Akt. Science 278: 687-689

32. Scheid MP, Schubert KM and Duronio V (1999) Regulation of bad phosphorylation and association with $\mathrm{Bcl}-\mathrm{x}(\mathrm{L})$ by the MAPK/Erk kinase. J. Biol. Chem. 274: 31108-31113

33. Cardone M, Roy N, Stennicke H, Salvesen G, Franke T, Stanbridge E, Frisch S and Reed J (1998) Regulation of cell death protease caspase-9 by phosphorylation. Science 282: 1318-1321

34. Allan L, Morrice N, Brady S, Magee G, Pathak S and Clarke P (2003) Inhibition of caspase-9 through phosphorylation at Thr 125 by ERK MAPK. Nat. Cell Biol. 5: $647-654$

35. Hernandez-Hoyos G, Sohn S, Rothenberg E and Alberola-lla J (2000) Lck activity controls CD4/CD8 T cell lineage commitment. Immunity 12: 313-322

36. Bommhardt U, Cole MS, Tso JY and Zamoyska R (1997) Signals through CD8 or CD4 can induce commitment to the CD4 lineage in the thymus. Eur. J. Immunol. 27: 1152-1163

37. Bommhardt U, Basson MA, Krummrei U and Zamoyska R (1999) Activation of the extracellular signal-related kinase/mitogen-activated protein kinase pathway discriminates CD4 versus CD8 lineage commitment in the thymus. J. Immunol. 163: 715-722

38. Li J, Sensebe L, Herve P and Charbord P (1997) Nontransformed colonyderived stromal cell lines from normal human marrows. III. The maintenance of hematopoiesis from CD34+ cell populations. Exp. Hematol. 25: 582-591

39. Ferrero I, Anjuere F, MacDonald $H$ and Ardavin C (1997) In vitro negative selection of viral superantigen-reactive thymocytes by thymic dendritic cells. Blood 90: 1943-1951

40. Zeira M, Gallily R, Stein I and Giloh H (1991) Thymocyte maturation following interaction with thymus-derived macrophages. Cell Immunol. 134: 370-377

41. Revy P, Sospedra M, Barbour B and Trautmann A (2001) Functional antigenindependent synapses formed between $\mathrm{T}$ cells and dendritic cells. Nat Immunol. 2: 925-931 